\title{
A Dirac Delta Operator
}

\author{
Juan Carlos Ferrando
}

Centro de Investigacion Operativa, Universidad Miguel Hernandez, E-03202 Elche, Spain

Cite This Paper in the following Citation Styles

(a): [1] Juan Carlos Ferrando, ”A Dirac Delta Operator," Mathematics and Statistics, Vol.9, No.2, pp. 179-187, 2021. DOI: 10.13189/ms.2021.090213.

(b): Juan Carlos Ferrando, (2021). A Dirac Delta Operator. Mathematics and Statistics, 9(2), 179-187. DOI: 10.13189/ms.2021.090213.

Copyright $\odot 2021$ by authors, all rights reserved. Authors agree that this article remains permanently open access under the terms of the Creative Commons Attribution License 4.0 International License

\begin{abstract}
If $T$ is a (densely defined) self-adjoint operator acting on a complex Hilbert space $\mathcal{H}$ and $I$ stands for the identity operator, we introduce the delta function operator $\lambda \mapsto \delta(\lambda I-T)$ at $T$. When $T$ is a bounded operator, then $\delta(\lambda I-T)$ is an operator-valued distribution. If $T$ is unbounded, $\delta(\lambda I-T)$ is a more general object that still retains some properties of distributions. We provide an explicit representation of $\delta(\lambda I-T)$ in some particular cases, derive various operative formulas involving $\delta(\lambda I-T)$ and give several applications of its usage in Spectral Theory as well as in Quantum Mechanics.
\end{abstract}

Keywords Hilbert Space, Self-adjoint Operator, Vectorvalued Distribution, Spectral Measure

\section{The delta function $\delta(\lambda I-T)$}

The scalar delta 'function' $\lambda \mapsto \delta(\lambda-a)$ along with its derivatives were introduced by Paul Dirac in [1], and later in [2, Section 15], although its definition can be traced back to Heaviside. The rigorous treatment of this object in the context of distribution theory is due to Laurent Schwartz [6, 12]. In this paper we extend the definition of $\delta(\lambda-a)$ from real numbers to self-adjoint operators on a Hilbert space $\mathcal{H}$. We denote by $\mathcal{D}(\mathbb{R})=\lim _{\mathcal{D}}([-n, n])$ the linear space of infinitely differentiable complex-valued functions of compact support, equipped with the inductive limit topology. As usual in physics we shall assume that the scalar product in $\mathcal{H}$ is anti-linear for the first variable.

If $T$ is a densely defined self-adjoint operator ${ }^{1}$ on $\mathcal{H}$ and $I$ stands for the identity operator, we define the delta function

\footnotetext{
${ }^{1}$ In what follows $\sigma(T)$ will denote the spectrum of $T$. Recall that the residual spectrum of a self-adjoint operator $T$ is empty, so that $\sigma(T)=$ $\sigma_{p}(T) \cup \sigma_{c}(T)$, where $\sigma_{p}(T)$ denotes the point spectrum (the eigenvalues) and $\sigma_{c}(T)$ the continuous spectrum of $T$.
}

operator $\lambda \mapsto \delta(\lambda I-T)$ at $T$ by

$$
f(T)=\int_{-\infty}^{+\infty} f(\lambda) \delta(\lambda I-T) d \lambda
$$

for each $f \in \mathcal{C}(\mathbb{R})$, i. e., for each real-valued continuous function $f(\lambda)$. Here $d \lambda$ is the Lebesgue measure of $\mathbb{R}$, but the right-hand side of (1.1) is not a true integral. If $T$ is a bounded operator, we shall see at once that $\delta(\lambda I-T)$ must be regarded as a vector-valued distribution, i. e., as a continuous linear map from the space $\mathcal{D}(\mathbb{R})$ into the locally convex space $\mathcal{L}(\mathcal{H})$ of the bounded linear operators (endomorphisms) on $\mathcal{H}$ equipped with the strong operator topology $[10,11]$, whose action on $f \in \mathcal{D}(\mathbb{R})$ we denote as an integral. If $T$ is unbounded we shall see that $\delta(\lambda I-T)$ still retains some useful distributional-like properties. The previous equation means

$$
\langle y, f(T) x\rangle=\int_{-\infty}^{+\infty} f(\lambda)\langle y, \delta(\lambda I-T) x\rangle d \lambda
$$

for each $(x, y) \in D(f(T)) \times \mathcal{H}$, where $D(f(T))$ stands for the domain of the self-adjoint operator $f(T)$.

Let us recall that if $T$ is a (densely defined) self-adjoint operator, there is a unique spectral family $\left\{E_{\lambda}: \lambda \in \mathbb{R}\right\}$ of selfadjoint operators defined on the whole of $\mathcal{H}$ that satisfy $(i)$ $E_{\lambda} \leq E_{\mu}$ and $E_{\lambda} E_{\mu}=E_{\lambda}$ for $\lambda \leq \mu$, (ii) $\lim _{\epsilon \rightarrow 0^{+}} E_{\lambda+\epsilon} x=$ $E_{\lambda} x$, and $($ iii $) \lim _{\lambda \rightarrow-\infty} E_{\lambda} x=\mathbf{0}$ and $\lim _{\lambda \rightarrow \infty} E_{\lambda} x=x$ in $\mathcal{H}$ for all $x \in \mathcal{H}$. The domain $D(T)$ of $T$ consists of those $x \in \mathcal{H}$ such that

$$
\int_{-\infty}^{+\infty}|\lambda|^{2} d\left\|E_{\lambda} x\right\|^{2}<\infty
$$

In this case, the spectral theorem (cf. [8, Section 107]) and the Borel-measurable functional calculus provide a self-adjoint operator $f(T)$ defined by

$$
f(T)=\int_{-\infty}^{+\infty} f(\lambda) d E_{\lambda}
$$


for each Borel-measurable function $f(\lambda)$, whose domain

$$
D(f(T))=\left\{x \in \mathcal{H}: \int_{-\infty}^{+\infty}|f(\lambda)|^{2} d\left\|E_{\lambda} x\right\|^{2}<\infty\right\}
$$

is dense in $\mathcal{H}$. Observe that if $T$ is bounded, $f(T)$ need not be bounded. Moreover, since $\lambda \mapsto E_{\lambda}$ is constant on the set $\mathbb{R} \backslash \sigma(T)$ of $T$, an open set in $\mathbb{R}$, equation (1.3) tell us that $f(\lambda)$ need not be defined on $\mathbb{R} \backslash \sigma(T)$.

Thanks to (1.3) the definition of $\delta(\lambda I-T)$ may be extended to Borel-measurable functions by declaring that the equation (1.1) holds for $(x, y) \in D(f(T)) \times \mathcal{H}$ and each Borel function $f$. But, by reasons that will become clear later, we shall restrict ourselves to those Borel functions which are continuous at each point of $\sigma_{p}(T)$. Moreover, working with the real and complex parts, no difficulty arises if the function $f$ involved in the equation (1.1) is complex-valued (except that $f(T)$ is no longer a self-adjoint operator whenever $\operatorname{Im} f \neq 0$ ). Thus, unless otherwise stated, we shall assume that both in (1.1) and (1.3) the function $f$ is complex-valued. Note that the complex Stieltjes measure $d\left\langle E_{\lambda} x, y\right\rangle$ need not be $d \lambda$-continuous. In what follows we shall denote by $\mathcal{B}_{p}(\mathbb{R})$ the linear space over $\mathbb{C}$ consisting of all complex-valued Borel-measurable functions of one real variable which are continuous on $\sigma_{p}(T)$.

If $f_{n} \rightarrow f$ in $\mathcal{D}(\mathbb{R})$, the sequence $\left\{f_{n}\right\}_{n=1}^{\infty}$ is uniformly bounded and $f_{n}(x) \rightarrow f(x)$ at each $x \in \mathbb{R}$. So, if $T$ is bounded on $\mathcal{H}$ (equivalently, self-adjoint on the whole of $\mathcal{H}$ ) it turns out that $f_{n}(T) \rightarrow f(T)$ in the strong operator topology [3, 10.2.8 Corollary]. Therefore, in this case $\delta(\lambda I-T)$ is an $\mathcal{L}(\mathcal{H})$-valued distribution.

As all integrals considered so far are over $\sigma(T)$, we have

$$
\delta(\lambda I-T)=\mathbf{0} \forall \lambda \notin \sigma(T) .
$$

Also $\delta(-\lambda I+T)=\delta(\lambda I-T)$ for all $\lambda \in \mathbb{R}$. On the other hand, if $\mu \in \sigma_{p}(T)$ and $y$ is an eigenvector corresponding to the eigenvalue $\mu$, clearly

$$
\delta(\lambda I-T) y=\delta(\lambda-\mu) y
$$

for every $\lambda \in \mathbb{R}$. In the particular case when $T_{a}$ is the linear operator defined on $\mathcal{H}$ by $T_{a} x=a x$ for a fixed $a \in \mathbb{R}$, then $T_{a}$ is a self-adjoint linear operator with $\sigma\left(T_{a}\right)=\sigma_{p}\left(T_{a}\right)=\{a\}$. In this case $\delta\left(\lambda I-T_{a}\right) x=\delta(\lambda-a) x$ for every $x \in \mathcal{H}$, i. e., $\delta\left(\lambda I-T_{a}\right)=\delta(\lambda-a) I$.

Since equality $\left\langle f(T)^{\dagger} y, x\right\rangle=\langle y, f(T) x\rangle$ holds for all $x, y \in D(f(T))$ and each $f \in \mathcal{B}_{p}(\mathbb{R})$, we may infer that

$$
\langle y, \delta(\lambda I-T) x\rangle=\langle\delta(\lambda I-T) y, x\rangle
$$

holds (in a 'distributional' sense) for all $x, y \in D(T)$. This suggests that in certain sense $\delta(\lambda I-T)$ may be regarded (possibly for almost all $\lambda \in \mathbb{R}$ ) as a Hermitian operator on $D(T)$.

Let us also point out that as equation (1.1) holds for all $f \in$ $\mathcal{D}(\mathbb{R})$, in a distributional sense we have

$$
\frac{d}{d \lambda}\left\langle y, E_{\lambda} x\right\rangle=\langle y, \delta(\lambda I-T) x\rangle
$$

If $\lambda \mapsto Y(\lambda-\mu)$ denotes the unit step function at $\mu \in \mathbb{R}$, given by $Y(\lambda-\mu)=0$ if $\lambda<\mu$ and $Y(\lambda-\mu)=1$ if $\lambda \geq \mu$, since $E_{\lambda}=Y(\lambda I-T)$ for each $\lambda \in \mathbb{R}$, formally

$$
d E_{\lambda} / d \lambda=Y^{\prime}(\lambda I-T)
$$

So, from (1.6) and (1.7) we get $Y^{\prime}(\lambda I-T)=\delta(\lambda I-T)$.

Proposition 1. If $T$ is a bounded self-adjoint operator on $\mathcal{H}$ and $f \in \mathcal{C}^{1}(\mathbb{R})$, then

$$
\int_{-\infty}^{+\infty} f(\lambda) \delta^{\prime}(\lambda I-T) d \lambda=-f^{\prime}(T) .
$$

The same equality holds if $T$ is unbounded but $f \in \mathcal{D}(\mathbb{R})$.

If $T$ is a self-adjoint operator and $f \in \mathcal{B}_{p}(\mathbb{R})$, then

$$
\int_{-\infty}^{+\infty}|f(\lambda)|^{2} \delta(\lambda I-T) d \lambda=\int_{-\infty}^{+\infty}|f(\lambda)|^{2} d E_{\lambda} .
$$

where the latter equality is the definition of $|f(T)|^{2}$. So, we have the following result.

Proposition 2. If $T$ is self-adjoint and $f \in \mathcal{B}_{p}(\mathbb{R})$, then

$$
\langle f(T) y, f(T) x\rangle=\int_{-\infty}^{+\infty}|f(\lambda)|^{2}\langle y, \delta(\lambda I-T) x\rangle d \lambda
$$

for every $x, y \in D(f(T))$.

Proof. We adapt a classic argument. Indeed, for every $x, y \in$ $D(f(T))$ we have

$$
\langle f(T) y, f(T) x\rangle=\int_{-\infty}^{+\infty} \overline{f(\lambda)} d\left\langle f(T) y, E_{\lambda} x\right\rangle .
$$

Since $E_{\mu} E_{\lambda}=E_{\mu}$ whenever $\mu \leq \lambda$, and $\left\langle E_{\lambda} y, x\right\rangle$ does not depend on $\mu$, by splitting the integral we get

$$
\int_{-\infty}^{+\infty} f(\mu) d\left\langle E_{\mu} y, E_{\lambda} x\right\rangle=\int_{-\infty}^{\lambda} f(\mu) d\left\langle y, E_{\mu} x\right\rangle,
$$

where clearly the first integral is $\left\langle f(T) y, E_{\lambda} x\right\rangle$. Plugging $d\left\langle f(T) y, E_{\lambda} x\right\rangle$ into (1.8), we are done.

Corollary 3. Under the same conditions of the previous theorem, the equality

$$
\|f(T) x\|^{2}=\int_{-\infty}^{+\infty}|f(\lambda)|^{2}\langle x, \delta(\lambda I-T) x\rangle d \lambda
$$

holds for every $x \in D(f(T))$.

Proposition 4. If $T$ is self-adjoint and $\left\{f_{n}\right\}_{n=1}^{\infty}$ is a uniformly bounded sequence in $\mathcal{B}_{p}(\mathbb{R})$ such that $f_{n} \rightarrow f$ pointwise on $\mathbb{R}$ with $f \in \mathcal{B}_{p}(\mathbb{R})$, then $f_{n}(T) x \rightarrow f(T) x$ for every $x \in$ $D(T)$.

Proof. This is a straightforward consequence of preceding corollary and the Lebesgue dominated convergence theorem. 
This proposition holds in particular if $f_{n} \rightarrow f$ in $\mathcal{D}(\mathbb{R})$. Hence, even in the unbounded case, $\delta(\lambda I-T)$ behaves as a vector-valued distribution-like object.

Proposition 5. Let $(\lambda, \mu) \mapsto g(\lambda, \mu)$ be a function defined on $\mathbb{R}^{2}$ such that $g(\lambda, \cdot) \in \mathcal{L}_{1}(\mathbb{R})$ for every $\lambda \in \mathbb{R}$ and $g(\cdot, \mu) \in$ $\mathcal{B}_{p}(\mathbb{R})$ for every $\mu \in \mathbb{R}$. If the parametric integral

$$
f(\lambda)=\int_{-\infty}^{+\infty} g(\lambda, \mu) d \mu
$$

is continuous on $\mathbb{R}$ and makes sense if we replace $\lambda$ by a selfadjoint operator $T$, the value of the integral

$$
\int_{-\infty}^{+\infty} \int_{-\infty}^{+\infty} g(\lambda, \mu) \delta(\lambda I-T) d \mu d \lambda
$$

does not depend on the integration ordering.

Proof. Since $g(\cdot, \mu) \in \mathcal{B}_{p}(\mathbb{R})$ for every $\mu \in \mathbb{R}$., one has

$$
g(T, \mu)=\int_{-\infty}^{+\infty} g(\lambda, \mu) \delta(\lambda I-T) d \lambda
$$

which implies

$$
f(T)=\int_{-\infty}^{+\infty}\left\{\int_{-\infty}^{+\infty} g(\lambda, \mu) \delta(\lambda I-T) d \lambda\right\} d \mu .
$$

On the other hand, by the definition of $\delta(\lambda I-T)$ we have

$$
f(T)=\int_{-\infty}^{+\infty}\left\{\int_{-\infty}^{+\infty} g(\lambda, \mu) d \mu\right\} \delta(\lambda I-T) d \lambda,
$$

for $(x, y) \in D(T) \times \mathcal{H}$. So, the proposition follows.

Theorem 6. If $T$ is a self-adjoint operator on $\mathcal{H}$, then

$$
\begin{gathered}
\int_{0}^{+\infty} f(\lambda) \delta\left(\lambda I-T^{2}\right) d \lambda= \\
\int_{0}^{+\infty} \frac{1}{2 \sqrt{\lambda}}\{\delta(\sqrt{\lambda} I-T)-\delta(\sqrt{\lambda} I+T)\} f(\lambda) d \lambda
\end{gathered}
$$

if $\lambda>0$ and $f \in \mathcal{B}_{p}(\mathbb{R})$, both members acting on $D\left(T^{2}\right)$.

Proof. First note that $T^{2} \geq 0$. Hence $\sigma\left(T^{2}\right) \subseteq[0,+\infty)$, which implies that $\delta\left(\lambda I-T^{2}\right)=\mathbf{0}$ if $\lambda<0$. Since $T^{2}$ is a self-adjoint operator, for $f \in \mathcal{B}_{p}(\mathbb{R})$ we have

$$
\int_{0}^{+\infty} f(\lambda) \delta\left(\lambda I-T^{2}\right) d \lambda=f\left(T^{2}\right)
$$

On the other hand, it is clear that

$\int_{0}^{+\infty} \frac{f(\lambda)}{2 \sqrt{\lambda}} \delta(\sqrt{\lambda} I-T) d \lambda=\int_{0}^{+\infty} f\left(\mu^{2}\right) \delta(\mu I-T) d \mu$

whereas, using that $\delta(-\mu I+T)=\delta(\mu I-T)$, we have

$-\int_{0}^{+\infty} \frac{f(\lambda)}{2 \sqrt{\lambda}} \delta(\sqrt{\lambda} I+T) d \lambda=\int_{-\infty}^{0} f\left(\mu^{2}\right) \delta(\mu I-T) d \mu$

So, the right-hand side of (1.10) coincides with

$$
\int_{-\infty}^{+\infty} f\left(\mu^{2}\right) \delta(\mu I-T) d \mu=f\left(T^{2}\right)
$$

since $\mu \mapsto f\left(\mu^{2}\right)$ is a Borel function.
If we denote by $L(\mathcal{H})$ the linear space of all linear endomorphisms on $\mathcal{H}$, the next theorem summarize some previous results.

Theorem 7. If $T$ is a densely defined self-adjoint operator on a Hilbert space $\mathcal{H}$, there is an $L(\mathcal{H})$-valued linear map $\delta_{T}$ on $\mathcal{B}_{p}(\mathbb{R})$, whose action on $f \in \mathcal{B}_{p}(\mathbb{R})$ we denote by

$$
\left\langle\delta_{T}, f\right\rangle=\int_{-\infty}^{+\infty} f(\lambda) \delta(\lambda I-T) d \lambda
$$

such that $\left\langle\delta_{T}, f\right\rangle=f(T)$. If $\left\{f_{n}\right\} \subseteq \mathcal{B}_{p}(\mathbb{R})$ is uniformly bounded and $f_{n}(t) \rightarrow f(t)$, with $f \in \mathcal{B}_{p}(\mathbb{R})$, for all $t \in \mathbb{R}$ then $\left\langle\delta_{T}, f_{n}\right\rangle x \rightarrow\left\langle\delta_{T}, f\right\rangle x$ for all $x \in \mathcal{H}$. If $T$ is bounded, $\delta_{T}$ is an $\mathcal{L}(\mathcal{H})$-valued distribution, so $\left\langle\delta_{T}, f\right\rangle$ is a bounded operator on $\mathcal{H}$. In addition $\delta(\lambda I-T)=\mathbf{0}$ if $\lambda \notin \sigma(T)$ and $\langle y, \delta(\lambda I-T) x\rangle=\langle\delta(\lambda I-T) y, x\rangle$ for $x, y \in D(T)$.

\section{Explicit form of $\delta(\lambda I-T)$}

If $Q$ is a vector-valued distribution, the Fourier transform of $Q$ is defined as the vector valued distribution $\mathcal{F} Q$ on $\mathcal{S}(\mathbb{R})$ such that $\langle\mathcal{F} Q, f\rangle=\langle Q, \mathcal{F} f\rangle$. As usual, we denote by $\mathcal{F}^{-1}$ the inverse Fourier transform.

Theorem 8. If $T$ is a self-adjoint operator, the identity

$$
\delta(\lambda I-T)=\frac{1}{2 \pi} \int_{-\infty}^{+\infty} e^{i t(\lambda I-T)} d t
$$

holds for every $\lambda \in \mathbb{R}$, and the action $f(T)$ of $\delta(\lambda I-T)$ on $f \in \mathcal{S}(\mathbb{R})$ is given by

$$
f(T)=\int_{-\infty}^{+\infty}\left\{\int_{-\infty}^{+\infty} \frac{f(\lambda)}{2 \pi} e^{i t(\lambda I-T)} d \lambda\right\} d t .
$$

Proof. Setting $\delta_{T}(\lambda)=\delta(\lambda I-T)$ observe that

$$
\left(\mathcal{F} \delta_{T}\right)(t)=\frac{1}{\sqrt{2 \pi}} e^{-i t T}
$$

Indeed, if $f \in \mathcal{S}(\mathbb{R})$ we have

$$
\begin{aligned}
\left\langle\mathcal{F} \delta_{T}, f\right\rangle & =\left\langle\delta_{T}, \mathcal{F} f\right\rangle=\int_{-\infty}^{+\infty}(\mathcal{F} f)(\lambda) \delta(\lambda I-T) d \lambda \\
& =(\mathcal{F} f)(T)=\frac{1}{\sqrt{2 \pi}} \int_{-\infty}^{+\infty} f(t) e^{-i t T} d t .
\end{aligned}
$$

Consequently

$$
\delta_{T}=\mathcal{F}^{-1}\left\{\frac{1}{\sqrt{2 \pi}} e^{-i t T}\right\}
$$

Functionally, the action of $\delta_{T}$ on $f \in S(\mathbb{R})$ by means of equation (2.2) becomes

$$
\left\langle\delta_{T}, f\right\rangle=\left\langle\frac{1}{\sqrt{2 \pi}} e^{-i t T},\left(\mathcal{F}^{-1} f\right)(t)\right\rangle
$$

Consequently, we have

$$
\left\langle\delta_{T}, f\right\rangle=\int_{-\infty}^{+\infty}\left\{\int_{-\infty}^{+\infty} \frac{f(\mu)}{2 \pi} e^{i t(\mu I-T)} d \mu\right\} d t
$$

with the order of the integration as stated. 
Corollary 9. If $e^{-i t T} x=x(t)$, for $x \in D(T)$ one has

$$
\delta(\lambda I-T) x=\frac{1}{2 \pi} \int_{-\infty}^{+\infty} e^{i \lambda t} x(t) d t
$$

and if $x \in D(f(T))$ and $f \in \mathcal{S}(\mathbb{R})$, then

$$
f(T) x=\frac{1}{2 \pi} \int_{-\infty}^{+\infty}\left\{\int_{-\infty}^{+\infty} f(\lambda) e^{i \lambda t} d \lambda\right\} x(t) d t .
$$

Remark 10. Consider the one-parameter unitary group $\{U(t): t \in \mathbb{R}\}$ generated by the self-adjoint operator $T$, that is, $U(t)=\exp (-i t T)$ for every $t \in \mathbb{R}$. If $\mathcal{F}$ denotes the Fourier transform, equation (2.2) can be written as

$$
\delta(\lambda I-T)=\frac{1}{\sqrt{2 \pi}} \mathcal{F}^{-1}(U)(\lambda) .
$$

So, equation (2.3) reads as

$$
f(T)=\frac{1}{\sqrt{2 \pi}} \int_{-\infty}^{+\infty}\left(\mathcal{F}^{-1} f\right)(t) U(t) d t .
$$

In what follows we shall compute the spectral family $\left\{E_{\lambda}: \lambda \in \mathbb{R}\right\}$ for some useful self-adjoint operators of Quantum Mechanics by means of the delta $\delta(\lambda I-T)$. Nonetheless, although $E_{\lambda}=Y(\lambda I-T)$, the identification

$$
Y(\lambda I-T)=\int_{-\infty}^{+\infty} Y(\lambda-\mu) \delta(\mu I-T) d \mu
$$

might be not well-defined because $\mu \mapsto Y(\lambda-\mu)$ has a jump discontinuity at $\mu=\lambda$. Indeed, if $\lambda \in \sigma_{p}(T)$ and $x$ is an eigenvector corresponding to $\lambda$, then

$\int_{-\infty}^{+\infty} Y(\lambda-\mu) \delta(\mu I-T) x d \mu=\left\{\int_{-\infty}^{\lambda} \delta(\mu-\lambda) d \mu\right\} x$

and the right-hand integral makes no sense (see [4] for a useful discussion). If $\lambda \notin \sigma_{p}(T)$ we define

$$
E_{\lambda}=\int_{-\infty}^{+\infty} Y(\lambda-\mu) \delta(\mu I-T) d \mu
$$

If $\lambda$ belongs to $\sigma_{p}(T)$, then $(\mu \mapsto Y(\lambda-\mu)) \notin \mathcal{B}_{p}(\mathbb{R})$. In order to define $E_{\lambda}$ we enlarge a little the interval of integration by considering the integral

$$
\int_{-\infty}^{\lambda+\epsilon} \delta(\mu-\lambda) d \mu
$$

for small $\epsilon>0$. So, if $\lambda \in \sigma_{p}(T)$ we define

$$
E_{\lambda}=\lim _{\epsilon \rightarrow 0^{+}} \int_{-\infty}^{+\infty} Y(\lambda+\epsilon-\mu) \delta(\mu I-T) d \mu .
$$

The limit is well-defined since $\lim _{\epsilon \rightarrow 0^{+}} E_{\lambda+\epsilon}=E_{\lambda}$ pointwise on $\mathcal{H}$. In the particular case when $\lambda$ belongs to $\sigma_{d}(T)$, the discrete part of $\sigma_{p}(T), \lambda$ is isolated in $\sigma_{p}(T)$.
Example 11. The spectral family of the (up to a sign) onedimensional Quantum Mechanics momentum operator of the free particle $P=i D$, where $D \varphi=\varphi^{\prime}$, acting on the Hilbert space $\mathcal{H}=L_{2}(\mathbb{R})$ is given by

$$
\left(E_{\lambda} \varphi\right)(x)=\frac{1}{2} \varphi(x)+\frac{1}{2 \pi i} \text { p.v. } \int_{-\infty}^{+\infty} \frac{e^{i \lambda(s-x)}}{s-x} \varphi(s) d s
$$

for every regular compactly supported $\varphi \in D(P)$.

Proof. As is well-known $P$ is a self-adjoint operator with $D(P)=H^{2,1}(\mathbb{R})$ and $\sigma_{c}(P)=\mathbb{R}$. Since

$$
\left(e^{-i t P} \varphi\right)(x)=\left(e^{t D} \varphi\right)(x)=\varphi(x+t)
$$

for a regular enough $\varphi \in D(P)$, by Corollary 9 we have

$$
\{\delta(\mu I-P) \varphi\}(x)=\frac{1}{2 \pi} \int_{-\infty}^{+\infty} e^{i \mu t} \varphi(x+t) d t .
$$

Note that the integral of the right-hand side does exist because $\varphi$ has compact support.

According to the definition of $E_{\lambda}$ for the continuous spectrum and keeping in mind the order of integration as indicated in Corollary 9, one has

$$
\left\{E_{\lambda} \varphi\right\}(x)=\frac{1}{2 \pi} \int_{-\infty}^{+\infty} \int_{-\infty}^{+\infty} Y(\lambda-\mu) e^{i \mu t} \varphi(x+t) d \mu d t .
$$

So, since

$$
\frac{1}{\sqrt{2 \pi}} \int_{-\infty}^{+\infty} Y(\lambda-\mu) e^{i \mu t} d \mu=\mathcal{F}(Y)(t) \cdot e^{i \lambda t},
$$

bearing in mind the distributional relation

$$
\mathcal{F}(Y)(t)=\sqrt{\frac{\pi}{2}}\left(\delta(t)+\frac{1}{i \pi} \mathrm{p} \cdot \mathrm{v} \cdot \frac{1}{t}\right),
$$

we get

$$
\left\{E_{\lambda} \varphi\right\}(x)=\frac{1}{2} \varphi(x)+\frac{1}{2 \pi i} \int_{-\infty}^{+\infty} \frac{e^{i \lambda(s-x)}}{s-x} \varphi(s) d s
$$

where the last integral must be understood in Cauchy's principal value sense.

Example 12. The spectral family of the one-dimensional Quantum Mechanics kinetic energy term of the free particle, corresponding to the Laplace operator $T=-D^{2}$ on $\mathcal{H}=$ $L_{2}(\mathbb{R})$, where $D^{2} \varphi=\varphi^{\prime \prime}$, is given by

$$
\left(E_{\lambda} \varphi\right)(x)=\frac{1}{i \pi} \mathrm{p} \cdot \mathrm{v} \cdot \int_{-\infty}^{+\infty} \frac{\cos (\lambda(s-x))-1}{s-x} \varphi(s) d s
$$

for $\lambda>0$ and $E_{\lambda}=\mathbf{0}$ whenever $\lambda<0$, where $\varphi$ is a regular function with compact support belonging to $D(T)$.

Proof. In this case $T$ is a self-adjoint operator with $\sigma(T)=$ $[0,+\infty)$. Since $T=(i D)^{2}$, according to (1.10) we have

$$
\delta(\lambda I-T)=\frac{1}{2 \sqrt{\lambda}}\{\delta(\sqrt{\lambda} I-i D)-\delta(\sqrt{\lambda} I+i D)\}
$$


regarded as a functional on $\mathcal{S}(\mathbb{R})$ through $d \lambda$-integration over $[0,+\infty)$. Plugging

$$
(\delta(\mu I \mp i D) \varphi)(x)=\frac{1}{2 \pi} \int_{-\infty}^{+\infty} e^{i \mu t} \varphi(x \pm t) d t
$$

into the previous expression and keeping in mind the correct order of integration, we see that

$$
\begin{gathered}
\int_{0}^{\infty} f(\lambda)(\delta(\lambda I-T) \varphi)(x) d \lambda= \\
\frac{1}{4 \pi} \int_{0}^{\infty} \int_{-\infty}^{+\infty} f(\lambda) \frac{e^{i \sqrt{\lambda} t}}{\sqrt{\lambda}}[\varphi(x+t)-\varphi(x-t)] d \lambda d t
\end{gathered}
$$

for every $f \in \mathcal{S}(\mathbb{R})$. By the definition of $E_{\lambda}$ if $\lambda>0$ and the fact that $\delta(\mu I-T)=\mathbf{0}$ whenever $\mu<0$, we have

$$
\left(E_{\lambda} \varphi\right)(x)=\int_{0}^{+\infty} Y(\lambda-\mu)(\delta(\mu I-T) \varphi)(x) d \mu .
$$

Working out the penultimate integral with $\mu$ instead of $\lambda$ and $f(\mu)=Y(\lambda-\mu)$, we obtain

$$
\begin{gathered}
\int_{0}^{+\infty} \int_{-\infty}^{+\infty} Y(\lambda-\mu) \frac{e^{i \sqrt{\mu} t}}{\sqrt{\mu}}[\varphi(x+t)-\varphi(x-t)] d \mu d t \\
=\int_{-\infty}^{+\infty}\left\{\int_{0}^{\lambda} \frac{e^{i \sqrt{\mu} t}}{\sqrt{\mu}} d \mu\right\}[\varphi(x+t)-\varphi(x-t)] d t
\end{gathered}
$$

for $\lambda>0$. So, by setting $u=\sqrt{\mu}$ we get

$$
\left(E_{\lambda} \varphi\right)(x)=\int_{-\infty}^{+\infty} \frac{d t}{2 \pi}[\varphi(x+t)-\varphi(x-t)] \int_{0}^{\lambda} e^{i u t} d u .
$$

Now we have

$$
\frac{1}{\sqrt{2 \pi}} \int_{0}^{\lambda} e^{i u t} d u=\left(1-e^{i \lambda t}\right) \mathcal{F}^{-1}(Y)(t)
$$

so, using that $\mathcal{F}^{-1}(Y(v))=\mathcal{F}(1-Y(v))(t)$ as well as equation (2.8), we get

$$
\frac{1}{\sqrt{2 \pi}} \int_{0}^{\lambda} e^{i u t} d u=\left(1-e^{i \lambda t}\right) \sqrt{\frac{\pi}{2}}\left(\delta(t)-\frac{1}{i \pi} \mathrm{p} \cdot \mathrm{v} \cdot \frac{1}{t}\right)
$$

which implies

$$
\begin{gathered}
\left(E_{\lambda} \varphi\right)(x)= \\
-\frac{1}{\pi i} \int_{-\infty}^{+\infty} \frac{\varphi(s)}{s-x} d s+\frac{1}{\pi i} \int_{-\infty}^{+\infty} \frac{\cos (\lambda(s-x))}{s-x} \varphi(s) d s
\end{gathered}
$$

where the integrals are understood in Cauchy's principal value sense.

Example 13. Spectral family of the (up to a sign) onedimensional Quantum Mechanics momentum operator $S$ for a bounded particle on $\mathcal{H}=L_{2}[-\pi, \pi]$ with domain

$$
\left\{\varphi \in L_{2}[-\pi, \pi]: \varphi^{\prime} \in L_{2}[-\pi, \pi], \varphi(-\pi)=\varphi(\pi)\right\}
$$

As is well-known this is a self-adjoint operator with discrete spectrum $\sigma(S)=\mathbb{Z}$ whose eigenfunction system $\left\{\varphi_{n}: n \in\right.$
$\mathbb{Z}$, with $\varphi_{n}(x)=(2 \pi)^{-1 / 2} e^{-i n x}$, are the solutions of the eigenvalue problem $i \varphi^{\prime}=\lambda \varphi$ with $\varphi(-\pi)=\varphi(\pi)$. So, for $\varphi \in D(S)$ we have $\varphi \stackrel{L_{2}}{=} \sum_{n \in \mathbb{Z}} c_{n} \varphi_{n}$ with

$$
c_{n}=\left\langle\varphi, \varphi_{n}\right\rangle=\frac{1}{2 \pi} \int_{-\pi}^{\pi} \varphi(x) e^{i n x} d x
$$

for every $n \in \mathbb{Z}$. Since $\sigma(S)=\sigma_{d}(S)$, recalling the definition of the operator $E_{\lambda}$ for $\lambda \in \sigma_{d}(S)$, clearly we have

$\left(E_{\lambda} \varphi\right)(x)=\lim _{\epsilon \rightarrow 0^{+}} \int_{-\infty}^{+\infty} Y(\lambda+\epsilon-\mu)(\delta(\mu I-S) \varphi)(x) d \mu$

for every $\lambda \in \mathbb{R}$. So, the fact that $E_{\lambda}$ is a bounded operator yields

$$
E_{\lambda} \varphi=\sum_{n \in \mathbb{Z}} c_{n} E_{\lambda} \varphi_{n}
$$

Using that $\delta(\mu I-S) e^{-i n x}=\delta(\mu-n) e^{-i n x}$ and that $Y(\lambda+0-n)=Y(\lambda-n)$, we get

$$
\left(E_{\lambda} \varphi\right)(x)=\sum_{n \in \mathbb{Z}} \frac{c_{n}}{\sqrt{2 \pi}} Y(\lambda-n) e^{-i n x}=\sum_{n \in \mathbb{Z}, n \leq[\lambda]} \frac{e^{-i n x}}{\sqrt{2 \pi}}
$$

Remark 14. Since in the previous example $S$ is bounded on $\mathcal{H}=L_{2}[-\pi, \pi]$, the delta operator $\delta(\lambda I-S)$ should be regarded as a continuous endomorphism as well. In this case

$$
\delta(\lambda I-S) \varphi=\sum_{n \in \mathbb{Z}} c_{n} \delta(\lambda-n) \varphi_{n}
$$

Example 15. The one-dimensional Quantum Mechanics position operator on $L_{2}(\mathbb{R})$. This operator is defined on $\mathcal{H}=$ $L_{2}(\mathbb{R})$ by $(Q \varphi)(x)=x \varphi(x)$ for every $x \in \mathbb{R}$. Clearly $\sigma_{c}(Q)=\mathbb{R}$ and $\varphi \in D(Q)$ if $(x \mapsto x \varphi(x)) \in \mathcal{L}_{2}(\mathbb{R})$. Moreover, it is clear that

$$
\{\exp (i t(\lambda I-Q)) \varphi\}(x)=e^{i(\lambda-x) t} \varphi(x) .
$$

So we have

$$
(\delta(\lambda I-Q) \varphi)(x)=\delta(\lambda-x) \varphi(x)
$$

Hence, in this case we can write

$$
\left\{E_{\lambda} \varphi\right\}(x)=\int_{-\infty}^{+\infty} Y(\lambda-\mu) \delta(\mu-x) \varphi(x) d \mu
$$

Therefore, if $\lambda \neq x$ we get

$$
\left\{E_{\lambda} \varphi\right\}(x)=Y(\lambda-x) \varphi(x) .
$$

Example 16. Explicit form of $\delta(\lambda I-M)$ for the Hermitian matrix of $\mathcal{H}=\mathbb{C}^{3}$

$$
M=\left[\begin{array}{lll}
0 & 1 & 1 \\
1 & 0 & 1 \\
1 & 1 & 0
\end{array}\right]
$$


Proof. In this case $M=P J_{M} P^{-1}$ with $\sigma(M)=\{-1,2\}$ and

$$
J_{M}=\left[\begin{array}{rrr}
-1 & 0 & 0 \\
0 & -1 & 0 \\
0 & 0 & 2
\end{array}\right], \quad P=\left[\begin{array}{rrr}
1 & 1 & 1 \\
-1 & 0 & 1 \\
0 & -1 & 1
\end{array}\right]
$$

Using (2.1) we get

$\delta(\lambda I-M)=P\left[\begin{array}{ccc}\delta(\lambda+1) & 0 & 0 \\ 0 & \delta(\lambda+1) & 0 \\ 0 & 0 & \delta(\lambda-2)\end{array}\right] P^{-1}$

Let us compute the spectral family and the projection operator onto the eigenspace $\operatorname{ker}(M+I)$. Clearly

$$
E_{\lambda}=P\left[\begin{array}{ccc}
Y(\lambda+1) & 0 & 0 \\
0 & Y(\lambda+1) & 0 \\
0 & 0 & Y(\lambda-2)
\end{array}\right] P^{-1}
$$

for every $\lambda \in \mathbb{R}$. If $\lambda_{1}=-1$, the orthogonal projection $P_{\lambda_{1}}$ onto $\operatorname{ker}(I+M)$ is

$$
P_{\lambda_{1}}=\frac{1}{3} P\left[\begin{array}{lll}
1 & 0 & 0 \\
0 & 1 & 0 \\
0 & 0 & 0
\end{array}\right] P^{-1}=\frac{1}{3}\left[\begin{array}{rrr}
2 & -1 & -1 \\
-1 & 2 & -1 \\
-1 & -1 & 2
\end{array}\right]
$$

since $P_{\lambda_{1}}=E_{\lambda_{1}}-E_{\lambda_{1}-0}=E_{\lambda_{1}}$.

Example 17. Consider a compact self-adjoint operator $K$ acting on a separable Hilbert space $\mathcal{H}$ which does not admit the eigenvalue zero. Let $\left\{u_{i}: i \in \mathbb{N}\right\}$ be a Hilbert basis of $\mathcal{H}$ with its corresponding sequence of real eigenvalues $\left\{\lambda_{i}: i \in \mathbb{N}\right\}$, where $\left|\lambda_{i+1}\right| \leq\left|\lambda_{i}\right|$ for every $i \in \mathbb{N}$. Let us compute the action of the operator $(\lambda I-K)^{-1}$ on any $x \in \mathcal{H}$ and the operator $\delta(\lambda I-T)$.

Proof. If $x \in \mathcal{H}$, we can write $x=\sum_{i=1}^{\infty}\left\langle x, u_{i}\right\rangle u_{i}$. Since $(\lambda I-K)^{-1}$ is a bounded operator whenever $\lambda \notin \sigma(K)$, we have

$$
(\lambda I-K)^{-1} x=\sum_{i=1}^{\infty}\left\langle x, u_{i}\right\rangle \int_{-\infty}^{+\infty} \frac{1}{\lambda-\mu} \delta(\mu I-K) u_{i} d \mu
$$

so we obtain the classic series

$$
(\lambda I-K)^{-1} x=\sum_{i=1}^{\infty} \frac{1}{\lambda-\mu_{i}}\left\langle x, u_{i}\right\rangle u_{i} .
$$

For the solution of the equation $(I-z K) x=y$ with $z \in \mathbb{C}$ we get the Schmidt series

$$
x=(I-z K)^{-1} y=\sum_{i=1}^{\infty} \frac{1}{1-z \mu_{i}}\left\langle y, u_{i}\right\rangle u_{i}
$$

whenever $z^{-1} \notin \sigma(T)$. On the other hand, since $\delta(\lambda I-K)$ acts on $\mathcal{H}$ as a continuous endomorphism, equation

$$
\delta(\lambda I-K) x=\sum_{i=1}^{\infty}\left\langle x, u_{i}\right\rangle \delta\left(\lambda-\mu_{i}\right) u_{i} .
$$

holds for every $x \in \mathcal{H}$.
If $T$ is an unbounded self-adjoint operator then $D(T) \neq \mathcal{H}$ and $D\left(T^{n}\right)$ becomes smaller as $n$ grows. So, the following result, makes sense only if the operator $T$ is bounded.

Theorem 18. In general, if $T$ is a bounded self-adjoint operator, one has

$$
\delta(\lambda I-T)=\sum_{n=0}^{\infty}(-1)^{n} \frac{\delta^{(n)}(\lambda)}{n !} T^{n}
$$

which is the Taylor series of $\delta(\lambda I-T)$ at $\lambda I$.

Proof. Developing the operator function $\exp (i t T)$, which is well-defined by the spectral theorem, we get

$$
\delta(\lambda I-T)=\frac{1}{2 \pi} \int_{-\infty}^{+\infty} e^{-i \lambda t} \sum_{n=0}^{\infty} \frac{(i t)^{n}}{n !} T^{n} d t,
$$

so that, formally interchanging the sum and the integral, we may write

$$
\delta(\lambda I-T)=\frac{1}{\sqrt{2 \pi}} \sum_{n=0}^{\infty} \frac{\mathcal{F}\left\{(i t)^{n}\right\}(\lambda)}{n !} T^{n} .
$$

Using the fact that

$$
\mathcal{F}\left\{(i t)^{n}\right\}(\lambda)=(-1)^{n} \sqrt{2 \pi} \delta^{(n)}(\lambda)
$$

for every $n \in \mathbb{N}$, we obtain (2.9).

\section{The resolvent operator

$$
\delta(\lambda I-T)
$$

Recall that the spectrum $\sigma(T)$ of a (densely defined) selfadjoint operator on a complex Hilbert space $\mathcal{H}$ is a closed subset of $\mathbb{C}$ contained in $\mathbb{R}$ (see for instance [9, 3.2]). If $z \in \mathbb{C} \backslash \sigma(T)$, i. e., if $z$ is a regular point of $T$, and

$$
\mathcal{R}(z, T)=(z I-T)^{-1}
$$

denotes the resolvent operator of $T$ at $z$ (see [7, Definition 8.2]), the function $\lambda \mapsto(z-\lambda)^{-1}$ is continuous on $\sigma(T)$. The resolvent is well-defined over $\mathcal{H}$, so it is a bounded normal operator. If $z \in \mathbb{R} \backslash \sigma(T)$ then $\mathcal{R}(z, T)$ is even self-adjoint. From (1.1) it follows that

$$
\mathcal{R}(z, T)=\int_{-\infty}^{+\infty} \frac{1}{z-\lambda} \delta(\lambda I-T) d \lambda
$$

which is the integral form of the resolvent of $T$. So, by considering the complex-valued function $f(\lambda)=(z-\lambda)^{-1}$ with $z \in \mathbb{C} \backslash \sigma(T)$ and using the fact that

$$
\mathcal{F}^{-1}\left(\frac{1}{\lambda-z}\right)(t)=\sqrt{2 \pi} i e^{i z t} Y(t)
$$

then, according to (2.5), for $\operatorname{Im} z>0$ we have

$$
(z I-T)^{-1}=-i \int_{0}^{\infty} e^{i z t} U(t) d t .
$$


From here, it follows that

$$
\mathcal{R}(z, i T)=i \mathcal{R}(i z,-T)=\left(\mathcal{L} U^{-1}\right)(z)
$$

if $\operatorname{Im} z>0$, where $\mathcal{L}$ is the Laplace transform. This is the Hille-Yosida theorem which relates the resolvent with the oneparameter group of unitary transformations $\{U(t): t \in \mathbb{R}\}$ generated by the self-adjoint operator $T$.

If $T$ is a bounded self-adjoint operator, $\gamma$ is a closed Jordan contour that encloses $\sigma(T)$ and $f(z)$ is holomorphic inside the connected region surrounded by the path $\gamma$, the Dunford integral formula asserts that

$$
\frac{1}{2 \pi i} \int_{\gamma} f(z) \mathcal{R}(z, T) d z=f(T) .
$$

In [13] is pointed out that $(2 \pi i)^{-1} \mathcal{R}(z, T)$ can be considered as the indicatrix of a vector-valued distribution with values in $\mathcal{L}(\mathcal{H})$. Dunford integral formula is easily obtained by using the $\delta(\lambda I-T)$ operator since, if we apply the Proposition 5 with $g(\lambda, \mu)=f(z(\mu))(z(\mu)-\lambda)^{-1}$, where $z(\mu)=\gamma(\mu)$ and $0 \leq \mu \leq 1$, then

$$
\begin{gathered}
\int_{\gamma} f(z) \mathcal{R}(z, T) d z=\int_{-\infty}^{+\infty}\left\{\int_{\gamma} \frac{f(z)}{z-\lambda} d z\right\} \delta(\lambda I-T) d \lambda \\
=2 \pi i \int_{-\infty}^{+\infty} f(\lambda) \delta(\lambda I-T) d \lambda=2 \pi i f(T) .
\end{gathered}
$$

Example 19. Derivation of the orthogonal projection operator onto $\operatorname{ker}(M+I)$ of the Hermitian matrix $M$ of the Example 16 by the resolvent technique. We must compute

$$
P_{\lambda_{1}}=\frac{1}{2 \pi i} \int_{|z+1|=1} \mathcal{R}(z, M) d z .
$$

Clearly, we have

$$
\mathcal{R}(z, M)=\frac{1}{z^{2}-z-2}\left[\begin{array}{ccc}
z-1 & 1 & 1 \\
1 & z-1 & 1 \\
1 & 1 & z-1
\end{array}\right] .
$$

Using that

$$
\int_{|z+1|=1} \frac{\{1, z-1\}}{(z+1)(z-2)} d z=\left\{-\frac{2 \pi i}{3}, \frac{4 \pi i}{3}\right\}
$$

we reproduce the result we got earlier.

\section{The $\delta(\lambda I-T)$ operator as a limit}

As $\mu \mapsto(\lambda \pm i \epsilon-\mu)^{-1}$ is continuous, for self-adjoint $T$

$$
\begin{gathered}
((\lambda-i \epsilon) I-T)^{-1}-((\lambda+i \epsilon) I-T)^{-1} \\
=\int_{-\infty}^{+\infty}\left(\frac{1}{\lambda-i \epsilon-\mu}-\frac{1}{\lambda+i \epsilon-\mu}\right) \delta(\mu I-T) d \mu .
\end{gathered}
$$

If $f \in \mathcal{D}(\mathbb{R})$, Proposition 5 yields

$$
\begin{aligned}
& \int_{-\infty}^{+\infty} \frac{f(\lambda)}{2 \pi i}\left\{((\lambda-i \epsilon) I-T)^{-1}-((\lambda+i \epsilon) I-T)^{-1}\right\} d \lambda \\
& =\frac{1}{\pi} \int_{-\infty}^{+\infty}\left\{\int_{-\infty}^{+\infty} f(\lambda) \frac{\epsilon}{(\lambda-\mu)^{2}+\epsilon^{2}} d \lambda\right\} \delta(\mu I-T) d \mu .
\end{aligned}
$$

Since in the sense of distributions

$$
\frac{1}{2 \pi i}\left(\frac{1}{\lambda-i \epsilon-\mu}-\frac{1}{\lambda+i \epsilon-\mu}\right) \rightarrow \delta(\lambda-\mu)
$$

as $\epsilon \rightarrow 0^{+}$, we have

$$
\frac{1}{\pi} \int_{\mathbb{R}} f(\lambda) \frac{\epsilon}{(\lambda-\mu)^{2}+\epsilon^{2}} d \lambda \rightarrow \int_{\mathbb{R}} f(\lambda) \delta(\lambda-\mu) d \lambda
$$

as $\epsilon \rightarrow 0^{+}$. Hence, if $g_{n}$ is defined by the left-hand side $\mu$-parametric integral with $\epsilon=1 / n$, then $g_{n} \rightarrow f$ pointwise on $\mathbb{R}$. So, if $f \in \mathcal{D}(\mathbb{R})$ and $T$ is bounded (hence with $\sigma(T)$ compact), as can be easily checked $\left\{g_{n}\right\}_{n=1}^{\infty}$ is a uniformly bounded sequence of continuous functions, with $\sup _{n \in \mathbb{N}}\left\|g_{n}\right\|_{\infty} \leq\|f\|_{\infty}$, that converges pointwise on $\mathbb{R}$ to $f$. Thus, by $\left[3,10.2 .8\right.$ Corollary] one has $g_{n}(T) \rightarrow f(T)$ in the strong operator topology, that is

$$
\begin{gathered}
\frac{1}{\pi} \int_{-\infty}^{+\infty}\left\{\int_{-\infty}^{+\infty} f(\lambda) \frac{\epsilon}{(\lambda-\mu)^{2}+\epsilon^{2}} d \lambda\right\} \delta(\mu I-T) d \mu \\
\rightarrow \int_{-\infty}^{+\infty} f(\mu) \delta(\mu I-T) d \mu
\end{gathered}
$$

as $\epsilon \rightarrow 0^{+}$in the strong operator topology of $\mathcal{L}(\mathcal{H})$. Therefore, if $T$ is bounded and $f \in \mathcal{D}(T)$ then

$$
\int_{-\infty}^{+\infty} \frac{f(\lambda)}{2 \pi i}\left\{((\lambda-i \epsilon) I-T)^{-1}-((\lambda+i \epsilon) I-T)^{-1}\right\} d \lambda
$$

goes to $f(T)$ as $\epsilon \rightarrow 0^{+}$. This proves that for bounded $T$

$$
\lim _{\epsilon \rightarrow 0^{+}} \frac{1}{2 \pi i}((\lambda-i \epsilon) I-T)^{-1}-((\lambda+i \epsilon) I-T)^{-1}
$$

coincides with $\delta(\lambda I-T)$ as an $\mathcal{L}(\mathcal{H})$-valued distribution.

\section{Unitary equivalence of $\delta(\lambda I-T)$}

Theorem 20. If $T$ is a self-adjoint operator defined on the whole of $\mathcal{H}$, there exist a finite measure $\mu$ on the Borel sets of the compact space $\sigma(T)$ and a linear isometry $U$ from $L_{2}(\sigma(T), \mu)$ onto $\mathcal{H}$ such that

$$
U^{-1} \delta(\lambda I-T) U=\delta(\lambda I-Q)
$$

where $(Q \varphi)(x)=x \varphi(x)$ is the position operator.

Proof. According to [5] there exist a finite measure $\mu$ on the Borel sets of the compact space $\sigma(T)$ and a linear isometry $U$ from $L_{2}(\sigma(T), \mu)$ onto $\mathcal{H}$ such that

$$
\left(U^{-1} T U\right) \varphi=Q \varphi
$$

for every $\varphi \in \mathcal{L}_{2}(\sigma(T), \mu)$. So, since $U^{-1} T U$ is a selfadjoint operator on $L_{2}(\sigma(T), \mu)$, we have

$$
U^{-1} \delta(\lambda I-T) U=\delta\left(\lambda I-U^{-1} T U\right)=\delta(\lambda I-Q)
$$

as stated.

Remark 21. For such linear isometry $U$ the equation

$$
\left(U^{-1} \delta(\lambda I-T) U \varphi\right)(x)=\delta(\lambda-x) \varphi(x)
$$

holds for every $\varphi \in \mathcal{L}_{2}(\sigma(T), \mu)$. 


\section{Commutation relations}

Let $S$ and $T$ be two self-adjoint operators defined on the whole of $\mathcal{H}$ for which equations $[S,[S, T]]=[T,[S, T]]=\mathbf{0}$ hold. In this case

$$
[-i t S,[-i t S,-i s T]]=i t^{2} s[S,[S, T]]=\mathbf{0}
$$

and the Baker-Campbell-Hausdorff formula yields

$$
\begin{gathered}
\delta(\lambda I-S) \delta(\mu I-T)= \\
\frac{1}{(2 \pi)^{2}} \iint_{\mathbb{R}^{2}} e^{i(t \lambda+s \mu)} e^{-i t S} e^{-i s T} d t d s= \\
\frac{1}{(2 \pi)^{2}} \iint_{\mathbb{R}^{2}} e^{i(t \lambda+s \mu)} e^{\frac{-s t}{2}[S, T]} e^{-i(t S+s T)} d t d s .
\end{gathered}
$$

Likewise, since $[T,[T, S]]=[S,[T, S]]=\mathbf{0}$ one has

$$
\begin{gathered}
\delta(\mu I-T) \delta(\lambda I-S)= \\
\frac{1}{(2 \pi)^{2}} \iint_{\mathbb{R}^{2}} e^{i(t \lambda+s \mu)} e^{\frac{-s t}{2}[T, S]} e^{-i(t T+s S)} d t d s \\
=\frac{1}{(2 \pi)^{2}} \iint_{\mathbb{R}^{2}} e^{i(t \lambda+s \mu)} e^{\frac{s t}{2}[S, T]} e^{-i(t S+s T)} d t d s .
\end{gathered}
$$

So, using that

$\exp \left(\frac{i s t}{2} i[S, T]\right)-\exp \left(-\frac{i s t}{2} i[S, T]\right)=2 i \sin \left(\frac{i s t}{2}[S, T]\right)$

we have

$$
\begin{gathered}
{[\delta(\lambda I-S), \delta(\mu I-T)]=} \\
\frac{i}{2 \pi^{2}} \iint_{\mathbb{R}^{2}} \sin \left(\frac{i s t}{2}[S, T]\right) e^{i(t \lambda+s \mu)} e^{-i(t S+s T)} d t d s .
\end{gathered}
$$

For position $Q$ and momentum $P$ of a one-dimensional particle, one has $\mathcal{H}=L_{2}(\mathbb{R})$ and $[Q, P]=i \hbar I$. Therefore $[Q,[Q, P]]=[P,[Q, P]]=\mathbf{0}$ and

$$
\begin{gathered}
{[\delta(\lambda I-Q), \delta(\mu I-P)]=} \\
\frac{i}{2 \pi^{2}} \iint_{\mathbb{R}^{2}} \sin \left(-\frac{s t}{2} \hbar\right) e^{i(t \lambda+s \mu)} e^{-i(t Q+s P)} d t d s .
\end{gathered}
$$

According to Theorem 8 , if $[\delta(\lambda I-S), \delta(\mu I-T)]$ acts on $f(\lambda)=\lambda$, formally we have

$$
\begin{gathered}
\int_{-\infty}^{+\infty} \lambda[\delta(\lambda I-S), \delta(\mu I-T)] d \lambda= \\
\frac{i}{2 \pi^{2}} \iint_{\mathbb{R}^{2}} \sin \left(\frac{i s t}{2}[S, T]\right) \\
\left\{\int_{-\infty}^{+\infty} \lambda e^{i t \lambda} d \lambda\right\} e^{i s \mu} e^{-i(t S+s T)} d t d s .
\end{gathered}
$$

So, using the distributional equality

$$
\int_{-\infty}^{+\infty} \lambda e^{i t \lambda} d \lambda=\frac{2 \pi}{i} \delta^{\prime}(t)
$$

and integrating by parts, it follows that

$$
\begin{aligned}
& \int_{-\infty}^{+\infty} \lambda[\delta(\lambda I-S), \delta(\mu I-T)] d \lambda \\
& =-\frac{i}{2 \pi}[S, T] \int_{-\infty}^{+\infty} s e^{i s(\mu I-T)} d s .
\end{aligned}
$$

Observe that a second application of equation (6.1) and a second integration by parts yield

$$
\begin{gathered}
\int_{-\infty}^{+\infty} \lambda \mu[\delta(\lambda I-S), \delta(\mu I-T)] d \lambda d \mu= \\
-\frac{i}{2 \pi}[S, T] \int_{-\infty}^{+\infty} s e^{-i s T}\left\{\int_{-\infty}^{+\infty} \mu e^{i s \mu} d \mu\right\} d s= \\
{[S, T] \int_{-\infty}^{+\infty} \delta(s)\{1-i s T\} e^{-i s T} d s=[S, T]}
\end{gathered}
$$

as expected.

\section{A remark on the Stone formula}

Let $T$ be a self-adjoint operator densely defined on a Hilbert space $\mathcal{H}$. If $A$ is a Borel set in $\sigma(T)$, defining

$$
E(A):=\int_{-\infty}^{+\infty} \chi_{A}(\lambda) d E_{\lambda}
$$

where $\chi_{A}$ stands for the characteristic function of $A$ (which is a bounded Borel function), then $E$ is an $\mathcal{L}(\mathcal{H})$-valued finitely additive and pointwise countably additive measure (i.e., countable additivity under the strong operator topology of $\mathcal{L}(\mathcal{H})$ ) on the $\sigma$-algebra $\mathcal{A}$ of Borel subsets of $\sigma(T)$. So, if the characteristic function $\chi_{A}$ of $A$ with respect to $\mathbb{R}$ is continuous on $\sigma_{p}(T)$ then

$$
E(A)=\int_{-\infty}^{+\infty} \chi_{A}(\lambda) \delta(\lambda I-T) d \lambda .
$$

For $-\infty<a<b<\infty$ and $\epsilon>0$, we have

$$
\begin{gathered}
\int_{a}^{b}\left\{\int_{-\infty}^{+\infty}\left(\frac{1}{\lambda-i \epsilon-\mu}-\frac{1}{\lambda+i \epsilon-\mu}\right) \delta(\mu I-T) d \mu\right\} d \lambda \\
=\int_{-\infty}^{+\infty}\left\{\int_{a}^{b} \frac{2 i \epsilon d \lambda}{(\lambda-\mu)^{2}+\epsilon^{2}}\right\} \delta(\mu I-T) d \mu= \\
2 i \int_{\mathbb{R}}\left\{\arg \tan \left(\frac{b-\mu}{\epsilon}\right)-\operatorname{argtan}\left(\frac{a-\mu}{\epsilon}\right)\right\} \delta(\mu I-T) d \mu .
\end{gathered}
$$

If the limit as $\epsilon \rightarrow 0^{+}$the bracketed function is equal to 0 if $\mu \in \mathbb{R} \backslash[a, b]$, equal to $\pi$ if $a<\mu<b$ and equal to $\pi / 2$ if $\mu \in\{a, b\}$. So, if $a, b \notin \sigma_{p}(T)$ so that $\chi_{(a, b)}$ and $\chi_{[a, b]}$ both belong to $\mathcal{B}_{p}(\mathbb{R})$, setting

$$
g_{n}(\mu):=\frac{1}{\pi} \int_{a}^{b} \frac{2 i n^{-1} d \lambda}{(\lambda-\mu)^{2}+n^{-2}}
$$

for each $n \in \mathbb{N}$ and

$$
f(\mu):=\chi_{(a, b)}(\mu)+\chi_{[a, b]}(\mu),
$$


then $g_{n}(\mu) \rightarrow f(\mu)$ for every $\mu \in \mathbb{R}$ and $\sup _{n \in \mathbb{N}}\left\|g_{n}\right\|_{\infty} \leq 1$ which, according to Proposition 4 , implies that $g_{n}(T) x \rightarrow$ $f(T) x$ for every $x \in D(T)$. In other words

$$
\begin{gathered}
\lim _{\epsilon \rightarrow 0^{+}} \frac{1}{2 \pi i} \int_{a}^{b}\left((\lambda-i \epsilon-T)^{-1}-(\lambda+i \epsilon-T)^{-1}\right) d \lambda \\
=\frac{1}{2} \int_{-\infty}^{+\infty}\left(\chi_{(a, b)}+\chi_{[a, b]}\right) \delta(\mu I-T) d \mu,
\end{gathered}
$$

holds pointwise on the domain $D(T)$ of $T$. Hence, by virtue of (7.1) we get

$$
\begin{aligned}
& \lim _{\epsilon \rightarrow 0^{+}} \frac{1}{2 \pi i} \int_{a}^{b}\left((\lambda-i \epsilon-T)^{-1}-(\lambda+i \epsilon-T)^{-1}\right) d \lambda= \\
& \frac{1}{2} E((a, b))+\frac{1}{2} E([a, b])=E(a, b)+\frac{1}{2} E(a)+\frac{1}{2} E(b)
\end{aligned}
$$

which is Stone's formula.

\section{REFERENCES}

[1] Dirac, P. A. M., The Physical Interpretation of the Quantum Dynamics, Proc. Royal Soc. London 113, 621-641, (1927).

DOI: $10.1098 /$ rspa.1927.0012

[2] Dirac, P. A. M., The Principles of Quantum Mechanics, 4th Edition, Clarendon Press, Oxford, 1988.

[3] Dunford, N. and Schwartz, J. T., Linear Operators. Part II: Spectral Theory, John Wiley, New York, 1988.

[4] Griffiths, D. and Walborn, S., Dirac deltas and discontinuous functions, Am. J. Phys. 67, 446-446, (1999).
DOI: $10.1119 / 1.19283$

[5] Halmos, P., What does the spectral theorem say? Amer. Math. Monthly 70, 241-247, (1963).

DOI: $10.1080 / 00029890.1963 .11990075$

[6] Horváth, J., Topological Vector Spaces and Distributions, Vol. I, Addison-Wesley, Reading, Massachusetts, 1966.

[7] Moretti, V., Spectral Theory and Quantum Mechanics, 2nd Edition, Unitext 110, Springer, 2013.

[8] Riesz, F., Nagy, B. Sz.-, Functional Analysis, Dover Publications, Mineola, 1990.

[9] Schmüdgen, K., Unbounded Self-adjoint Operators on Hilbert Space, Springer, Dordrecht, 2012.

[10] Schwartz, L., Théorie des distributions à valeurs vectorielles. I, Anal. Inst. Fourier, 1-141, 7 (1957).

DOI : 10.5802/aif.68

[11] Schwartz, L., Théorie des distributions à valeurs vectorielles. II, Anal. Inst. Fourier 8, 1-209, (1958).

DOI: 10.5802/aif.77

[12] Schwartz, L., Théorie des Distributions, Hermann, Paris, 1966.

[13] Tillmann, H. G., Vector-valued distributions and the spectral theorem for self-adjoint operators in Hilbert space, Bull. Amer. Math. Soc. 69, 67-71, (1963).

DOI: 10.1090/S0002-9904-1963-10862-9 